\title{
Ebola-are you at risk?
}

\section{Editorial}

The lethal Ebola virus had remained contained in one area in Africa before it began to move on a vector course to western Africa and possibly beyond. What can account for this migration of a relatively geographically static disease? People and animals such as monkeys carry this disease and both migrate for various reasons. Monkeys have market value and may be captured in one area and transported to another for transshipment to other countries carrying diseases with them. People have become more mobile than ever and disease can migrate at the speed of air travel. What is especially problematic is when a disease is initially asymptomatic and the carrier is relocating with the disease and does not know it. All countries should be prepared for an emerging viral attack by planning how they will handle an outbreak. This means modern equipment to quarantine patients and current training for health care workers to prevent them from contracting the disease. Recently two Americans were transferred to the U.S. for definitive treatment of their infection with Ebola because it was considered prudent since in the U.S. they would receive more modern medical care and the quarantine isolation facility would be more secure; however, this does not help the countries in western Africa who are still dealing with an epidemic of Ebola cases. They need the best in modern quarantine equipment and the best in medical equipment to treat their patients.

The problem with any emerging disease in developing countries is their lack of capacity to manage both the medical care of the infected and the prevention of additional infections. Fighting viruses takes technology, education and often sophisticated protective quarantine equipment. Each of these are often in short supply in developing countries and can even be taxing on the existing resources in developed countries depending on the magnitude of the spread of the epidemic and the number of people infected or at continuing risk for infection particularly health care workers and emergency first responders such as firefighters, paramedics and police officers who are called to an emergency that then ends up being a case of the viral disease but too late they are informed after they are infected and become patients themselves.

The only method of controlling Ebola at this time is containment in a quarantine environment where the patient is treated intensively and the health care workers are protected from accidental infection. Ebola will travel to new victims as people move; and they either know or don't know they are infected. Certainly, anyone who knows they are infected and has the financial resources to get to a developed country for definitive care, will be on the first flight out and potentially spread the disease as they travel to get advanced medical care in a modern, developed country. Economic development does not make anyone immune from contact infection and the disease spreads to countries other than the point of origin where the disease might be contained by geography or poverty meaning the infected population cannot travel and thus cannot spread the disease by casual contact with others.

A few countries, including the U.S. and other African countries now have Ebola patients in hospitals where they have never experienced Ebola before. If they remain vigilant, it is a risk but maybe not a
Volume 2 Issue 2 - 2015

\section{MichaelW Popejoy \\ 'Department of Public Health Nutrition, Zayed University, Dubai ${ }^{2}$ Faculty of Medicine, An-Najah National University, Palestine} 3ackson State University, USA

Correspondence: Michael W Popejoy,Adjunct Professor of Public Health, Department of Health Promotion and Disease Prevention, Robert Stempel School of Public Health and Social Work, Florida International University, Miami, Florida, USA, Tel 5613732267, Email dr_popejoy@hotmail.com

Received: March 12, 2015 | Published: March 17, 2015

sure thing that the disease will escape the quarantine to infect others particularly health care workers and laboratory researchers. However; if Ebola is in country, the risk is always present that it will begin its movement in a country that has never before experienced the disease. In other words, the Ebola outbreak has already broken out of its geographical containment hot zone which is ground zero as it is now on the move as people and certain animals move. The question we must ask as we face this epidemic is - is it wise to move Ebola patients to modern medical facilities in countries where the disease is not endemic or move modern medical equipment and containment technology to the countries where the disease is now ground zero hot zone? Some report that the risk of infection is countries like the U.S. is low as long as the patients are handled properly; however, the risk of the disease breaking out remains a reality. Maybe the medical teams in developed countries such as the U.S. may be too overconfident in their ability to contain this dangerous virus without significant risk of an outbreak here.

A critical problem in the U.S. where we have not really learned our lesson from the major epidemic of Spanish flu in 1918 that killed an estimated 100million people globally is that government and public health are not fully integrated sufficiently to enable a rapid and effective response to mitigating another massive epidemic. The Ebola situation, although not resulting in widespread disease in America due to its contact transmission rather than airborne transmission, certainly demonstrated that our own head of the U.S. Center for Disease Control and Prevention (CDC) does not have the power to call for a localized or generalized quarantine; and our government officials such as the President and state governors lacked the political will or understanding of risk to call for a quarantine under their executive authority. Unfortunately, we may face a future epidemic that will not allow sufficient time for our characteristic slow response and it will be a disaster to a national population left unprotected by either 
government or public health due to their inability to work together with the urgency necessitated by the emergency facing the nation. I wonder how many other nations have similar disconnects between their governments and their public health scientists and physicians. Most national governments must soon revise their public policies on how they will handle a pandemic disease attack within their borders.

\section{Acknowledgements}

None.

\section{Conflict of interest}

The author declares no conflict of interest. 